Pacific Journal of Mathematics

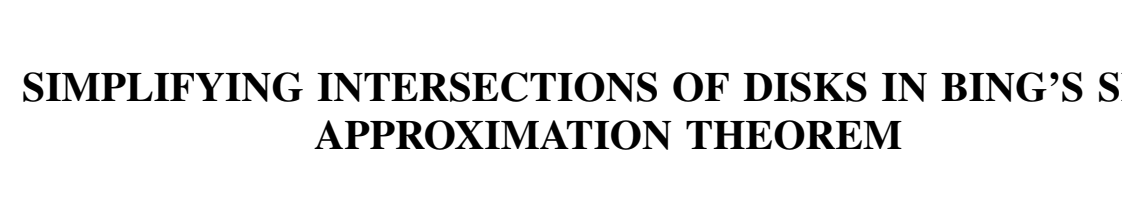




\title{
SIMPLIFYING INTERSECTIONS OF DISKS IN BING'S SIDE APPROXIMATION THEOREM
}

\author{
F. M. LISTER
}

In Bing's Side Approximation Theorem for 2-spheres in $E^{3}$ the disks on the approximating sphere and the disks on the given sphere may intersect in a very complicated manner. It is shown in this paper that the disks may be chosen so that there are the same number of disks on the approximating sphere as on the given sphere and the disks intersect in a one-to-one fashion. Furthermore, the approximating homeomorphism may be chosen so that it maps each disk on the given sphere onto the disk on the approximating sphere which it intersects.

Applications are given to a study of the preservation of tameness of subsets of the boundary of a crumpled cube under re-embeddings of the crumpled cube in $E^{3}$.

An $\varepsilon$-mapping of a subset of $E^{3}$ into $E^{3}$ is a mapping which moves no point a distance as much as $\varepsilon$. An $\varepsilon$-set in $E^{3}$ is a set of diameter less than $\varepsilon$. David Gillman [6] and L. D. Loveland [10, 11] have used the following definition in connection with tame subsets of 2-spheres in $E^{3}$.

Property $(*, F, V)$. If $F$ is a closed subset of a 2-sphere $S$ in $E^{3}$ and $V$ is a component of $E^{3}-S$, then $(*, F, V)$ is satisfied if for each $\varepsilon>0$ there exist an $\varepsilon$-homeomorphism $h$ of $S$ into $E^{3}$, a finite collection of mutually exclusive $\varepsilon$-disks $\left\{D_{1}, D_{2}, \cdots, D_{r}\right\}$ on $h(s)$, and a finite collection of mutually exclusive $\varepsilon$-disks $\left\{E_{1}, E_{2}, \cdots, E_{n}\right\}$ on $S$ such that

(i) $h(s)$ is polyhedral,

(ii ) $h(s)-U$ Int $D_{j} \subset V$,

(iii) $h(s) \cap s \subset \bigcup$ Int $E_{i}$, and

(iv) $\left(\bigcup E_{i}\right) \cap F=\varnothing$.

Bing's Side Approximation Theorem [3, Th. 16] may then be stated as follows.

Theorem A (Bing). If $S$ is a 2-sphere in $E^{3}$ and $V$ is a component of $E^{3}-S$, then $(*, \varnothing, V)$ is satisfied.

We show in $\S 6$ that if $(*, F, V)$ is satisfied, then the homeomorphism $h$ and the collections of disks may be chosen so that the following additional properties are satisfied.

( v ) $r=n$, 
(vi) $h\left(E_{i}\right)=D_{i}$, and

(vii) $D_{i} \cap S=h(s) \cap E_{i}=\left(\right.$ Int $\left.D_{i}\right) \cap\left(\operatorname{Int} E_{i}\right)$.

We denote the property with conditions (i)-(vii) by $(* *, F, V)$. Thus, as shown by Theorem $2,(*, F, V)$ is equivalent to $(* *, F, V)$. This strengthens Theorem A, that is, $(* *, \varnothing, V)$ holds for any 2-sphere $S$ and either complementary domain $V$ in $E^{3}$. Furthermore, it is a consequence of the Tietze Extension Theorem [8, page 82] and Dehn's Lemma [12] that if $(* *, F, V)$ is satisfied and $g$ is a homeomorphism of $S \cup V$ into $E^{3}$, then $(* *, g(F), g(V))$ is satisfied. The same invariance then also holds for $(*, F, V)$. See $\S 9$. Some theorems on the invariance of tameness and half tameness (as defined below) of subsets of a 2-sphere $S$ in $E^{3}$ under homeomorphisms of $S \cup V$ into $E^{3}$ follow from this result and are given in $\S 10$.

Most of the definitions in this paper are like those used by Loveland in [10] and [11]. We say that a closed subset $F$ of a 2-sphere in $E^{3}$ is half tame if it is a subset of some 2-sphere which is tame from one of its complementary domains and that $F$ is tame if it is a subset of a tame 2 -sphere.

To show that $(*, F, V)$ implies $(* *, F, V)$ we begin with an approximation to $S$ from $V$ of the type given by $(*, F, V)$. Then by a series of steps involving a new selection of disks at each step we obtain a finite collection of mutually exclusive small disks $\left\{D_{1}, D_{2}, \cdots, D_{n}\right\}$ on the approximating sphere $h(S)$ in one-to-one correspondence with a collection of $n$ mutually exclusive small disks $\left\{E_{1}, E_{2}, \cdots, E_{n}\right\}$ on $S$ with the property that $h(S)-\bigcup \operatorname{Int} D_{i} \subset V$, the closure of the component of $D_{i}-S$ containing $\mathrm{Bd} D_{i}$ intersects $S$ in the interior of the corresponding disk $E_{i}$, and $\left(\bigcup E_{i}\right) \cap F=\varnothing$. The proof is then completed with an application of the Tietze Extension Theorem, Dehn's Lemma, and a homeomorphism of the approximating sphere onto itself. Details are given in Sections 4, 5, and 6 .

2. Covering collections of disks. This section includes a key step in obtaining the new collection of mutually exclusive small disks on the approximating sphere. This result is stated and proved as Lemma 3 below. The following lemma is a theorem of plane topology and is stated here without proof.

Lemma 1. If $\left\{K_{1}, K_{2}, \cdots, K_{n}\right\}$ is a finite collection of mutually exclusive compact sets in $E^{2}$ such that $E^{2}-U K_{i}$ is connected, then there is a collection of mutually exclusive disks $\left\{D_{1}, D_{3}, \cdots, D_{n}\right\}$ in $E^{2}$ such that $K_{i} \subset \operatorname{Int} D_{i}$.

A special cellular decomposition of a 2-sphere $S$ is a finite cellular decomposition of $S$ in which each pair of intersecting cells intersect 
in an arc. Each 2-sphere has a special cellular decomposition of arbitrarily small mesh [2]. The star st $C_{p}$ of a cell $C_{p}$ of a cellular decomposition is the union of the cells which intersect $C_{p}$. If $\mathscr{A}$ is a collection of sets we denote the union of the sets in $\mathscr{A}$ by $\mathscr{A}^{*}$.

Lemma 2. If $\mathscr{C}=\left\{C_{1}, C_{2}, \cdots, C_{m}\right\}$ is a special cellular decomposition of a 2 -sphere $S, \mathscr{D}$ is a finite collection of mutually exclusive disks each of which is a subset of the interior of a cell of $\mathscr{C}$, and $\left\{\mathscr{D}_{1}, \mathscr{D}_{2}, \cdots, \mathscr{D}_{m}\right\}$ is a partition of $\mathscr{D}$ (plus possibly some empty collections) such that $\mathscr{D}_{p}^{*} \subset$ st $C_{p}(p=1,2, \cdots, m)$, then there is a collection of mutually exclusive disks $\left\{D_{1}, D_{2}, \cdots, D_{m}\right\}$ such that $\mathscr{D}_{p}^{*} \subset \operatorname{Int} D_{p} \subset$ st $C_{p}$.

Proof. Without loss of generality we assume that for each $p, \mathscr{D}_{p}$ is nonempty and there is a disk of $\mathscr{D}_{p}$ in each cell of the star of $C_{p}$. Denote the subcollection of $\mathscr{D}_{q}$ each disk of which lies in $C_{p}$ by $\mathscr{D}_{q p}$. Now use Lemma 1 to cover each $\mathscr{D}_{q p}^{*}$ by a disk $D_{q p}$ which lies in $C_{p}$ in such a way that the disks in the resulting collection are mutually exclusive. Then there is exactly one disk in Int $C_{p}$ associated with each cell in st $C_{p}$. $A$ pattern like that in Figure 1 is obtained. For $q \neq p$ there is an arc $A_{q p}$ from $D_{q p}$ to $C_{q}$ which lies except for end points in (Int $C_{p}-\cup D_{r p}$ ) such that the arcs in the resulting collection are mutually exclusive. Next there is an arc $B_{q p}$ from $D_{q q}$ to the end point of the arc $A_{q p}$ such that $B_{q p}$ lies, except for its intersection

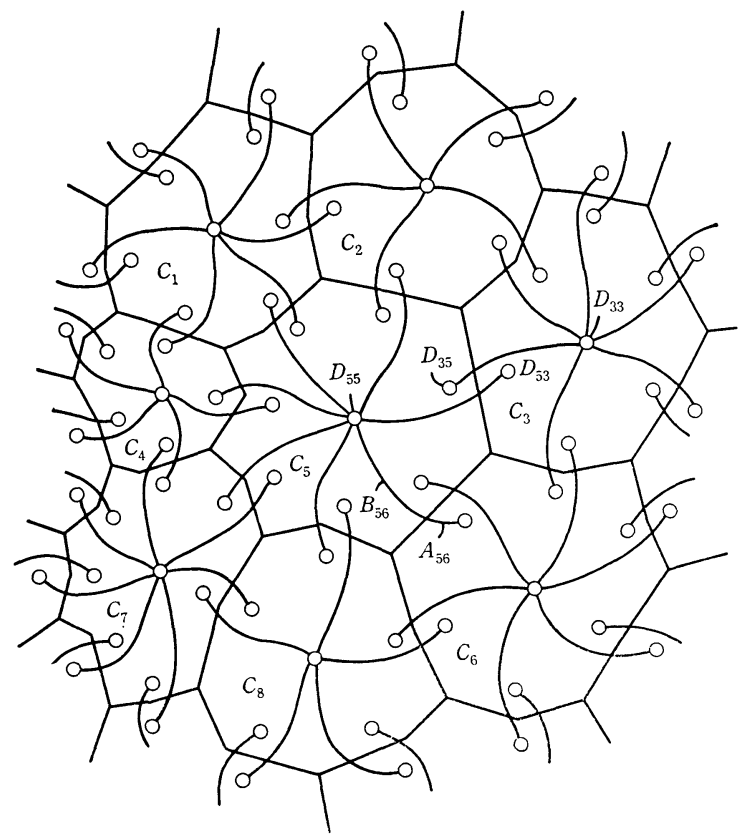

Figure 1. 
with $A_{q p}$, in (Int $C_{q}-\bigcup D_{r q}-\bigcup A_{s q}$ ). The arcs in the resulting collection $\left\{A_{q p} \cup B_{q p}\right\}$ over all of $S$ are mutually exclusive. The required disks are obtained (from the spider-like figures in Figure 1) by replacing each of these arcs by a thin disk.

Lemma 3. If $\mathscr{C}=\left\{C_{1}, C_{2}, \cdots, C_{m}\right\}$ is a special cellular decomposition of a 2-sphere $S, \mathscr{D}$ is a finite collection of mutually exclusive disks each of which is a subset of the interior of a cell of $\mathscr{C}$, and $\left\{\mathscr{D}_{1}, \mathscr{D}_{2}, \cdots, \mathscr{D}_{n}\right\}$ is a partition of $\mathscr{D}$ such that $\mathscr{D}_{i}{ }^{*} \subset$ st $C_{p}$ for some $C_{p} \in \mathscr{C}$, then there is a collection of mutually exclusive disks $\left\{D_{1}, D_{2}, \cdots, D_{n}\right\}$ such that $\mathscr{D}_{i}^{*} \subset \operatorname{Int} D_{i} \subset$ st $C_{q}$ for some $C_{q} \in \mathscr{C}$.

Proof. Assign each subcollection $\mathscr{D}_{i}$ a cell $C_{p(i)}$ of $\mathscr{C}$ such that $\mathscr{D}_{i}{ }^{*} \subset \operatorname{st} C_{p(i)}$. Let $\mathscr{E}_{p}$ be the union of all $\mathscr{D}_{i}^{\prime} s$ assigned to $C_{p}$. Then $\left\{\mathscr{E}_{1}, \mathscr{E}_{2}, \cdots, \mathscr{E}_{m}\right\}$ is a partition of $\mathscr{D}$ (plus possibly some empty collections) satisfying the hypothesis of Lemma 2. Hence there is a collection of mutually exclusive disks $\left\{E_{1}, E_{2}, \cdots, E_{m}\right\}$ such that $\mathscr{E}_{p}^{*} \subset \operatorname{Int} E_{p} \subset$ st $C_{p}$. Now use Lemma 1 to cover each $\mathscr{D}_{i}^{*}$ in Int $E_{p}$ by a disk $D_{i}$ in Int $E_{p}$ such that the disks in the resulting collection are mutually exclusive. Then $\left\{D_{1}, D_{2}, \cdots, D_{n}\right\}$ is a collection of disks satisfying the conclusion of Lemma 3 .

For a given collection $\mathscr{D}$ of mutually exclusive disks on $S$ to satisfy the hypothesis of Lemma 3 that each disk be contained in the interior of a cell of the cellular decomposition, it may be necessary to obtain a new cellular decomposition by a homeomorphism of $S$ onto itself which carries the 1-skeleton of a given cellular decomposition off the disks of $\mathscr{D}$. The procedure is like that used by Bing in [2] and we simply state the following lemma for future use.

Lemma 4. If $K$ is the 1-skeleton of a cellular decomposition of a 2-sphere $S, \varepsilon>0$, and $\left\{D_{1}, D_{2}, \cdots, D_{n}\right\}$ is a finite collection of mutually exclusive $\varepsilon$-disks on $S$, then there is an $\varepsilon$-homeomorphism $h$ of $S$ onto itself such that $\left(\bigcup D_{i}\right) \cap h(K)=\varnothing$.

3. Intersection of a disk with a finite collection of disks. Another important step in obtaining a new collection of disks on the approximating sphere is given in this section. The main result is Lemma 6 , but the following lemma is needed first.

Lemma 5. If $S$ is a 2-sphere in $E^{3},\left\{E_{1}, E_{2}, \cdots, E_{n}\right\}$ is a collection of mutually exclusive disks on $S, V$ is a component of $E^{3}-S, D$ is a disk in $E^{3}$ with $\mathrm{Bd} D$ contained in $V$, and $G$ is the component of $D-S$ containing $\mathrm{Bd} D$ such that $(\mathrm{cl} G) \cap S=(\mathrm{cl} G) \cap\left(\mathrm{U} \operatorname{Int} E_{i}\right)$ and 
$(\mathrm{cl} G) \cap E_{i} \neq \varnothing(i=1,2, \cdots, n)$ then the union of any collection of components of $D-G$ fails to separate Int $D$ and each component of $D-G$ intersects exactly one of the sets $(\operatorname{cl} G) \cap E_{i}(i=1,2, \cdots, n)$.

Proof. Let $K=D-G$. For each component $K^{\alpha}$ of $K$, $K^{\alpha} \cap(\mathrm{cl} G-\mathrm{Bd} D) \neq \varnothing$ and $G-\mathrm{Bd} D$ is connected. It follows that if $\mathscr{A}$ is an indexing set for the set of components of $K$ and $\mathscr{B} \subset \mathscr{A}$ then Int $D-\bigcup_{\alpha \in \mathscr{B}} K^{\alpha}=(G-\operatorname{Bd} D) \cup \bigcup_{\alpha \in \mathscr{A} \rightarrow \mathscr{A}} K^{\alpha}$ is connected. It follows from the fact that spheres are unicoherent [13, page 60] that $K^{\alpha} \cap \mathrm{cl}\left(\right.$ Int $\left.D-K^{\alpha}\right)$ is connected for each $\alpha \in \mathscr{A}$ and therefore $K^{\alpha}$ may intersect at most one of the sets $(\mathrm{cl} G) \cap E_{i}$. Since it must intersect at least one of these sets, $K^{\alpha}$ intersects exactly one.

Lemma 6. If the hypothesis of Lemma 5 is satisfied, then there is a collection of mutually exclusive disks $\left\{D_{1}, D_{2}, \cdots, D_{n}\right\}$ in $\operatorname{Int} D$ such that $\mathrm{Bd} D_{i} \subset V$ and $\left(\mathrm{cl} G_{i}\right) \cap S=\left(\operatorname{cl} G_{i}\right) \cap \operatorname{Int} E_{i}=(\mathrm{cl} G) \cap \operatorname{Int} E_{i}$ $(i=1,2, \cdots, n)$, where $G_{i}$ is the component of $D_{i}-S$ containing $\operatorname{Bd} D_{i}$.

Proof. By Lemma 5 each component of $D-S$ intersects exactly one of the sets $(\mathrm{cl} G) \cap E_{i}$. Let $\mathscr{K}_{i}$ be the collection of all components of $D-G$ which intersect $(\mathrm{cl} G) \cap E_{i}$. By Lemma $5, \mathscr{K}_{i}^{*}$ fails to separate Int $D$. Since the $E_{i}^{\prime}$ 's are mutually exclusive, $\left\{\mathscr{K}_{1}^{*}, \mathscr{K}_{2}^{*}\right.$, $\left.\cdots, \mathscr{K}_{n}^{*}\right\}$ is a collection of sets satisfying the hypothesis of Lemma 1. Hence there is a collection of mutually exclusive disks $\left\{D_{1}, D_{2}\right.$, $\left.\cdots, D_{n}\right\}$ in Int $D$ such that $\mathscr{K}_{i}^{*} \subset \operatorname{Int} D_{i}$. Note that $(\operatorname{cl} G) \cap E_{i}=$ (cl $G) \cap \mathscr{K}_{i}^{*}$ and (cl $\left.G\right) \cap E_{i}$ fails to intersect $E_{k}$ for $k \neq i$. Thus each $D_{i}$ has the property that $\mathrm{Bd} D_{i} \subset V$ and $\left(\operatorname{cl~} G_{i}\right) \cap S=\left(\operatorname{cl} G_{i}\right) \cap \operatorname{Int} E_{i}=$ $(\operatorname{cl} G) \cap \operatorname{Int} E_{i} . \quad D_{1}^{\prime},\left\{D_{11}, D_{12}\right\}, G_{1},\left\{G_{11}, G_{12}\right\}$ illustrate this in Figure 2.

4. Pairing disks in side approximations. The preceding lemmas along with the Tietze Extension Theorem and Dehn's Lemma are used to prove the following theorem which shows that a side approximation to a sphere may be replaced by another such that the disks intersect in a one-to-one fashion. See Figure 2.

THeOREM 1. If $F$ is a closed subset of a 2-sphere $S$ in $E^{3}, V$ is a component of $E^{3}-S$ such that $(*, F, V)$ is satisfied, and $\varepsilon>0$, then there exist an $\varepsilon$-homeomorphism $h$ of $S$ into $E^{3}$, a finite collection of mutually exclusive $\varepsilon$-disks $\left\{D_{1}, D_{2}, \cdots, D_{n}\right\}$ on $h(S)$, and a collection of $n$ mutually exclusive $\varepsilon$-disks $\left\{E_{1}, E_{2}, \cdots, E_{n}\right\}$ on $S$ such that

(i) $h(S)$ is polyhedral,

(ii) $h(S)-\bigcup \operatorname{Int} D_{i} \subset V$,

(iii) $D_{i} \cap S=h(S) \cap E_{i}=\left(\operatorname{Int} D_{i}\right) \cap\left(\operatorname{Int} E_{i}\right)$, and 

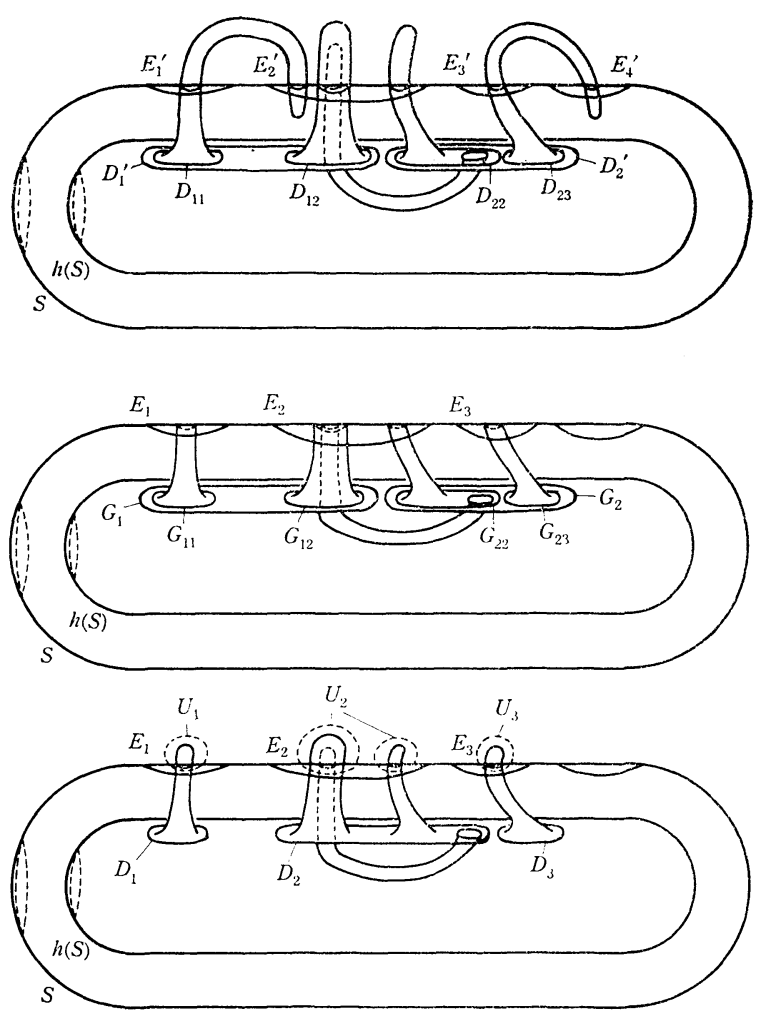

Figure 2.

(iv) $\left(\bigcup E_{i}\right) \cap F=\varnothing$.

Proof. Let $\mathscr{C}=\left\{C_{1}, C_{2}, \cdots, C_{m}\right\}$ be a special cellular decomposition of $S$ of mesh less than $\varepsilon / 9$, and let

$$
\begin{gathered}
\delta=\min \left\{1 / 3 \min \left\{\rho\left(C_{p}, C_{q}\right): C_{p} \cap C_{q}=\varnothing ; C_{p}, C_{q} \in \mathscr{C}\right\},\right. \\
\left.1 / 2 \min \left\{\varepsilon / 9-\operatorname{diam} C_{p}: C_{p} \in \mathscr{C}\right\}\right\} .
\end{gathered}
$$

By $(*, F, V)$ there exist a $\delta / 2$-homeomorphism $h_{1}$ of $S$ into $E^{3}$, a finite collection of mutually exclusive $\delta / 2$-disks $\left\{D_{1}^{\prime}, D_{2}^{\prime}, \cdots, D_{r}^{\prime}\right\}$ on $h_{1}(S)$, and a finite collection of mutually exclusive $\delta / 2$-disks $\left\{E_{1}^{\prime}, E_{2}^{\prime}, \cdots, E_{t}^{\prime}\right\}$ on $S$ such that

$$
h_{1}(S) \text { is polyhedral, }
$$

$$
h_{1}(S)-\bigcup \operatorname{Int} D_{j}^{\prime} \subset V,
$$

$$
h_{1}(S) \cap S=\left(\bigcup \operatorname{Int} D_{j}^{\prime}\right) \cap S \subset \bigcup \operatorname{Int} E_{i}^{\prime}, \quad \text { and }
$$

$\left(\bigcup E_{i}^{\prime}\right) \cap F=\varnothing$.

Note that $\mathrm{Bd} D_{j}^{\prime} \subset V$. If $\left(\bigcup \begin{array}{l}\prime \\ j\end{array}\right) \cap S=\varnothing$ we are finished. Otherwise 
let $G_{j}$ denote the component of $D_{j}^{\prime}-S$ containing $\mathrm{Bd} D_{j}^{\prime}$ and let $\left\{E_{1}, E_{2}, \cdots, E_{n}\right\}$ be the subcollection of $\left\{E_{1}^{\prime}, E_{2}^{\prime}, \cdots, E_{t}^{\prime}\right\}$ such that

$$
\left(\mathrm{cl} G_{j}\right) \cap E_{i} \neq \varnothing \text { for some } j(j=1,2, \cdots, r) \text {. }
$$

Then $\left\{E_{1}, E_{2}, \cdots, E_{n}\right\}$ is the collection of disks on $S$ in the conclusion of the theorem. Note that $\left(\bigcup E_{i}\right) \cap F=\varnothing$. The collection $\left\{D_{1}, D_{2}\right.$, $\left.\cdots, D_{n}\right\}$ remains to be identified.

Let $K$ be the 1 -skeleton of $\mathscr{C}$. By Lemma 4 there is a $\delta / 2$ homeomorphism $h_{2}$ of $h_{1}(S)$ onto itself such that

$$
\left(\bigcup D_{j}^{\prime}\right) \cap h_{2} h_{1}(K)=\varnothing \text {. }
$$

It follows that for each $j(j=1,2, \cdots, r)$

$$
D_{j}^{\prime} \subset \operatorname{Int} h_{2} h_{1}\left(C_{p}\right) \text { for some } C_{p} \in \mathscr{C} .
$$

Let $g=h_{2} h_{1}$. Then $g$ is a $\delta$-homeomorphism. Suppose $x, y \in C_{p}$. Then $\rho(x, y) \leqq \operatorname{diam} C_{p}$ and it follows from (1) that

$$
\begin{aligned}
\rho(g(x), g(y)) \leqq & \rho(g(x), x)+\rho(x, y)+\rho(g(y), y) \\
< & 1 / 2\left(\varepsilon / 9-\operatorname{diam} C_{p}\right)+\operatorname{diam} C_{p} \\
& +1 / 2\left(\varepsilon / 9-\operatorname{diam} C_{p}\right) \\
= & \varepsilon / 9 .
\end{aligned}
$$

Hence

$$
\operatorname{diam} g\left(C_{p}\right)<\varepsilon / 9(p=1,2, \cdots, m) .
$$

Now suppose $g\left(C_{p}\right) \cap E_{i} \neq \varnothing$ and $g\left(C_{q}\right) \cap E_{i} \neq \varnothing$. Let $x \in g\left(C_{p}\right) \cap E_{i}$ and $y \in g\left(C_{q}\right) \cap E_{i}$. Note that $g^{-1}$ is a $\delta$-homeomorphism. Then by (1)

$$
\begin{aligned}
\rho\left(g^{-1}(x), g^{-1}(y)\right) & \leqq \rho\left(g^{-1}(x), x\right)+\rho(x, y)+\rho\left(g^{-1}(y), y\right) \\
& <\delta+\delta / 2+\delta \\
& <5 / 6 \min \left\{\rho\left(C_{r}, C_{s}\right): C_{r} \cap C_{s}=\varnothing ; C_{r}, C_{s} \in \mathscr{C}\right\} .
\end{aligned}
$$

Hence $C_{p} \cap C_{q} \neq \varnothing$ and therefore

$$
\begin{aligned}
& \text { if } g\left(C_{p}\right) \text { and } g\left(C_{q}\right) \text { intersect the same } E_{i} \text {, then } \\
& g\left(C_{p}\right) \text { and } g\left(C_{q}\right) \text { are identical or adjacent cells. }
\end{aligned}
$$

For each $j(j=1,2, \cdots, r)$, let $\mathscr{E}_{j}$ be the subcollection of $E_{i}$ 's such that $\left(\mathrm{cl} G_{j}\right) \cap E_{i} \neq \varnothing$. By (4) and (6),

$$
\left(\mathrm{cl} G_{j}\right) \cap S=\left(\operatorname{cl} G_{j}\right) \cap\left(\bigcup \operatorname{Int} E_{i}: E_{i} \in \mathscr{E}_{j}\right) .
$$

Hence the hypotheses of Lemma 5 and Lemma 6 are satisfied and as a consequence there is a collection of mutually exclusive disks $\left\{D_{j i}: i=\right.$ $j(1), j(2), \cdots, n(j)\}$ in Int $D_{j}^{\prime}$ such that $\operatorname{Bd} D_{j i} \subset V$ and $\left(\operatorname{cl} G_{j i}\right) \cap S=$ 
$\left(\operatorname{cl~} G_{j i}\right) \cap \operatorname{Int} E_{i}=\left(\operatorname{cl} G_{j}\right) \cap \operatorname{Int} E_{i}$ where $G_{j i}$ is the component of $D_{j i}-S$ containing $\mathrm{Bd} D_{j i}$. Note that $h(S)-\mathrm{U} \operatorname{Int} D_{j i} \subset V$.

Now for each fixed $i(i=1,2, \cdots, n)$, let $\mathscr{D}_{i}$ be the subcollection of $D_{j k}$ 's such that $\left(\mathrm{cl} G_{j k}\right) \cap E_{i} \neq \varnothing$. By (12) each $\mathscr{D}_{i}$ is contained in the star of a cell $g\left(C_{p}\right)$ for some $C_{p} \in \mathscr{C}$. Then $\left\{\mathscr{D}_{1}, \mathscr{D}_{2}, \cdots, \mathscr{D}_{n}\right\}$ is a family of collections of disks satisfying the hypothesis of Lemma 3. Hence there is a collection of mutually exclusive disks $\left\{D_{1}^{\prime \prime}, D_{2}^{\prime \prime}\right.$, $\left.\cdots, D_{n}^{\prime \prime}\right\}$ such that $\mathscr{D}_{i}^{*} \subset \operatorname{Int} D_{i}^{\prime \prime} \subset \operatorname{st} g\left(C_{p}\right)$. Note that $\operatorname{Bd} D_{i}^{\prime \prime} \subset V$. We may assume without loss of generality that each disk $D_{i}^{\prime \prime}$ has a polygonal boundary. Let $G_{i}^{\prime \prime}$ be the component of $D_{i}^{\prime \prime}-S$ containing Bd $D_{i}^{\prime \prime}$. Each $D_{i}^{\prime \prime}$ has the property that $\operatorname{cl} G_{i}^{\prime \prime}$ intersects $S$ in $\operatorname{Int} E_{i}$. It remains to replace each $D_{i}^{\prime \prime}$ by a disk $D_{i}$ which intersects $S$ in Int $E_{i}$ if it intersects $S$ at all.

By the Tietze Extension Theorem extend the identity map on $\left(\mathrm{cl} G_{i}^{\prime \prime}\right) \cap E_{i}$ to a map $f_{i}$ on $D_{i}^{\prime \prime}-G_{i}^{\prime \prime}$ into a subdisk $E_{i}^{\prime \prime}$ of Int $E_{i}$ which contains (cl $\left.G_{i}^{\prime \prime}\right) \cap E_{i}$. Then extend $f_{i}$ to $D_{i}^{\prime \prime}$ by defining it to be the identity on $G_{i}^{\prime \prime}$. Since $D_{i}^{\prime \prime} \subset \operatorname{st} g\left(C_{p}\right)$ and diam st $g\left(C_{r}\right)<\varepsilon / 3$ for each $C_{r} \in \mathscr{C}$, it follows that $\operatorname{diam} D_{i}^{\prime \prime}<\varepsilon / 3$. Also $\operatorname{diam} E_{i}<\varepsilon / 36$. Therefore for each $i(i=1,2, \cdots, n)$,

$$
\operatorname{diam} f_{i}\left(D_{i}^{\prime \prime}\right) \leqq \operatorname{diam} D_{i}^{\prime \prime}+\operatorname{Diam} E_{i}<13 \varepsilon / 36 .
$$

Let $\gamma_{i}=1 / 2\left(13 \varepsilon / 36-\operatorname{diam} f_{i}\left(D_{i}^{\prime \prime}\right)\right)$ and let $U_{i}$ be a $\gamma_{i}$-neighborhood of the set of singularities of $f_{i}\left(D_{i}^{\prime \prime}\right)$ such that

$$
\begin{gathered}
U_{i} \cap S \subset \operatorname{Int} E_{i}, \\
\left(U_{i} \cup f_{i}\left(D_{i}^{\prime \prime}\right)\right) \cap\left(U_{k} \cup f_{k}\left(D_{k}^{\prime \prime}\right)\right)=\varnothing \quad \text { for } i \neq k, \quad \text { and } \\
\left(\bigcup U_{i}\right) \cap\left(g(S)-\bigcup \operatorname{Int} D_{i}^{\prime \prime}\right)=\varnothing .
\end{gathered}
$$

By Dehn's Lemma there is for each $i(i=1,2, \cdots, n)$, a polyhedral disk $D_{i} \subset f_{i}\left(D_{i}^{\prime \prime}\right) \cup U_{i}$ with $\mathrm{Bd} D_{i}=\mathrm{Bd} D_{i}^{\prime \prime}$. By the way $U_{i}$ is defined, $\left(g(S)-\bigcup D_{i}^{\prime \prime}\right) \cup \cup D_{i}$ is a polyhedral 2-sphere, and for each $i$,

$$
\operatorname{diam} D_{i}<13 \varepsilon / 36 \text {. }
$$

Now define $h=g$ on $g^{-1}\left(g(S)-\bigcup D_{i}^{\prime \prime}\right)$ and as follows on $g^{-1}\left(\bigcup D_{i}^{\prime \prime}\right)$. First extend the identity map on $\mathrm{U} B d D_{i}^{\prime \prime}=\bigcup \mathrm{Ud} D_{i}$ to a homeomorphism $h^{\prime}$ of $\bigcup D_{i}^{\prime \prime}$ onto $\bigcup D_{i}$. Then for each $y \in \bigcup D_{i}^{\prime \prime}$,

$$
\begin{aligned}
\rho\left(h^{\prime}(y), y\right) & \leqq \max \left\{\operatorname{diam} D_{i}^{\prime \prime}+\operatorname{diam} D_{i}: i=1,2, \cdots, n\right\} \\
& <\varepsilon / 3+13 \varepsilon / 36=25 \varepsilon / 36 .
\end{aligned}
$$

Since $g$ is a $\delta$-homeomorphism,

$$
\rho(g(x), x)<\varepsilon / 18 \text { for each } x \in S .
$$


Let $h=h^{\prime} g$ on $g^{-1}\left(\bigcup \mathbf{U} D_{i}^{\prime \prime}\right)$. Then by (18) and (19),

$$
\rho(h(x), x)<25 \varepsilon / 36+\varepsilon / 18=3 \varepsilon / 4 \quad \text { on } g^{-1}\left(\bigcup D_{i}^{\prime \prime}\right) .
$$

Then $h$ is the required homeomorphism and $\left\{D_{1}, D_{2}, \cdots, D_{n}\right\}$ and $\left\{E_{1}, E_{2}, \cdots, E_{n}\right\}$ are the required collections of disks.

5. Pairing disks with a homeomorphism. In Lemma 10 we prove the main lemma needed to show that the approximating homeomorphism may be chosen so that it maps each disk on the given sphere onto the corresponding disk of the approximating sphere. The proofs of Lemma 7 and Lemma 8 below are straight-forward and are not given.

Lemma 7. If $D$ is a disk and $\left\{D_{1}, D_{2}, \cdots, D_{n}\right\} \cup\left\{E_{1}, E_{2}, \cdots, E_{n}\right\}$ is a collection of mutually exclusive disks in Int $D$, then there is a homeomorphism $h$ of $D$ onto itself which is the identity on $\operatorname{Bd} D$ and carries $E_{i}$ onto $D_{i}(i=1,2, \cdots, n)$.

Lemma 8. If $\left\{D_{1}, D_{2}, \cdots, D_{r}\right\}$ and $\left\{E_{1}, E_{2}, \cdots, E_{n}\right\}$ are finite collections of mutually exclusive disks in the interior of a disk $D$, then there is a homeomorphism $g$ of $D$ onto itself which is the identity

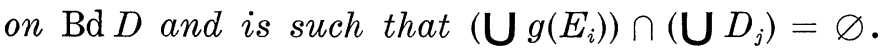

Lemma 9. If $\mathscr{C}$ is a special cellular decomposition of a 2-sphere $S,\left\{D_{1}, D_{2}, \cdots, D_{n}\right\} \cup\left\{E_{1}, E_{2}, \cdots, E_{n}\right\}$ is a collection of mutually exclusive disks with corresponding disks $D_{i}$ and $E_{i}$ in the interior of the same cell or in the interiors of adjacent cells of $\mathscr{C}$, then there is a homeomorphism $h$ of $S$ onto itself such that $h\left(E_{i}\right)=D_{i}$ and for each $x \in S, x$ and $h(x)$ are either in the same cell or in adjacent cells of $\mathscr{C}$.

Proof. For each $C_{p} \in \mathscr{C}$ cover all $D_{i}$ 's in $C_{p}$ which are associated with $E_{i}$ 's in the same cell $C_{q}$ (which must either be $C_{p}$ or a cell adjacent to $C_{p}$ ) by a disk $F_{p q}$ in Int $C_{p}$ and cover all $E_{i}$ 's in $C_{p}$ which correspond to $D_{i}$ 's in the same cell $C_{r}$ by a disk $H_{p r}$ in Int $C_{p}$ so that the disks in the total resulting collection are mutually exclusive. This may be done by Lemma 1 . There is an arc $A_{p q}$ from $F_{p q}$ to $H_{q p}$ such that $A_{p q}$ lies except for end points in $\left(C_{p} \cup C_{q}\right)-\left(\cup F_{p r} \cup \cup H_{q s}\right)$ and the arcs in the total resulting collection are mutually exclusive. See Figure 3. If these arcs are replaced by thin disks a collection of mutually exclusive disks $\left\{K_{p q}\right\}$ is obtained with $F_{p q} \cup H_{q p} \subset K_{p q}$. Now apply Lemma 7 to obtain a homeomorphism $h$ of $S$ onto itself such that $h\left(E_{i}\right)=D_{i}(i=1,2, \cdots, n)$.

Lemma 10. If $\mathscr{C}$ is a special cellular decomposition of a 2-sphere 


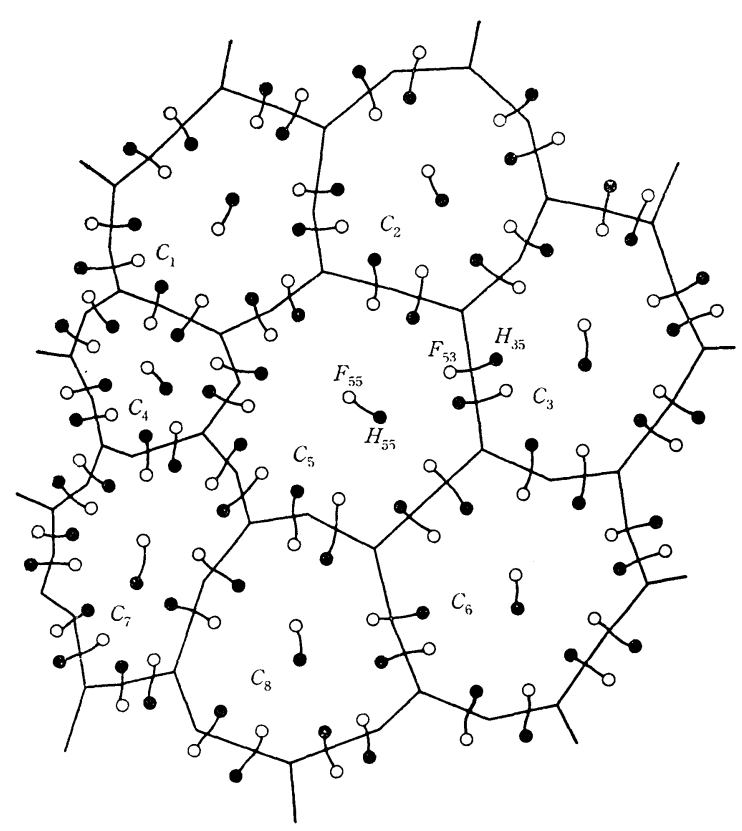

Figure 3.

$S,\left\{D_{1}, D_{2}, \cdots, D_{n}\right\}$ is a collection of mutually exclusive disks on $S,\left\{E_{1}, E_{2}, \cdots, E_{n}\right\}$ is a collection of mutually exclusive disks on $S$, and $D_{i}$ and $E_{i}$ are contained in the interior of the same cell or in the interiors of adjacent cells of $\mathscr{C}$, then there is a homeomorphism $h$ of $S$ onto itself such that $h\left(E_{i}\right)=D_{i}$ for each $i$, and for each $x \in S, x$ and $h(x)$ are either in the same cell or in adjacent cells of $\mathscr{C}$.

Proof. By Lemma 8 there is a homeomorphism $g$ of $S$ onto itself which is the identity on the 1-skeleton of $\mathscr{C}$ and sends each cell $C_{p}$ onto itself such that $\left(\bigcup g\left(E_{i}\right)\right) \cap\left(\bigcup D_{j}\right)=\varnothing$. By Lemma 9 there is a homeomorphism $f$ of $S$ onto itself such that $f g\left(E_{i}\right)=D_{i}$ and for each $x \in S, x$ and $f(x)$ are either in the same cell or in adjacent cells of $\mathscr{C}$. Then $h=f g$ is the required homeomorphism.

6. Equivalence of $(*, F, V)$ and $(* *, F, V)$. The lemmas of $\S 5$ are used to strengthen Theorem 1 by adding the condition that $h\left(E_{i}\right)=D_{i}$.

THeOREM 2. If $F$ is a closed subset of a 2-sphere $S$ in $E^{3}$ and $V$ is a component of $E^{3}-S$, then $(*, F, V)$ is satisfied if and only if $(* *, F, V)$ is satisfied.

Proof. It is clear that $(* *, F, V)$ implies $(*, F, V)$. To prove 
the converse let $\varepsilon>0$ and let $\mathscr{C}=\left\{C_{1}, C_{2}, \cdots, C_{m}\right\}$ be a special cellular decomposition of $S$ such that diam st $C_{p}<\varepsilon / 3$ for each $C_{p} \in \mathscr{C}$. Let

$$
\begin{array}{r}
\delta=1 / 4 \min \left\{\min \left\{\varepsilon / 3-\operatorname{diam~st~} C_{p}: C_{p} \in \mathscr{C}\right\},\right. \\
\left.\min \left\{\rho\left(C_{p}, S-\operatorname{st} C_{p}\right): C_{p} \in \mathscr{C}\right\}\right\} .
\end{array}
$$

By Theorem 1 there is a $\delta / 4$-homeomorphism $g$ of $S$ into $E^{3}$, a finite collection of mutually exclusive $\delta / 4$-disks $\left\{D_{1}, D_{2}, \cdots, D_{n}\right\}$ on $g(S)$ and a finite collection of mutually exclusive $\delta / 4$-disks $\left\{E_{1}, E_{2}, \cdots, E_{n}\right\}$ on $S$ such that

$$
\begin{gathered}
g(S) \text { is polyhedral }, \\
g(S)-\bigcup \operatorname{Int} D_{i} \subset V, \\
D_{i} \cap S=h(S) \cap E_{i}=\left(\operatorname{Int} D_{i}\right) \cap\left(\operatorname{Int} E_{i}\right), \quad \text { and } \\
\left(\bigcup E_{i}\right) \cap F=\varnothing .
\end{gathered}
$$

Without loss of generality assume that $\left(\operatorname{Int} D_{i}\right) \cap\left(\operatorname{Int} E_{i}\right) \neq \varnothing,(i=$ $1,2, \cdots, n)$. By Lemma 4 there is a $\delta / 4$-homeomorphism $h_{1}$ of $S$ onto itself such that $\left(\bigcup E_{i}\right) \cap h_{1}(K)=\varnothing$ where $K$ is the 1 -skeleton of $\mathscr{C}$. Similarly there is a $\delta / 4$-homeomorphism $h_{2}$ of $g(S)$ onto itself such that $\left(\bigcup D_{i}\right) \cap h_{2} g(K)=\varnothing$. Note that

$$
\begin{aligned}
& D_{i} \subset \operatorname{Int} h_{2} g\left(C_{q}\right) \text { for some } C_{q} \in \mathscr{C} \text {, } \\
& E_{i} \subset \operatorname{Int} h_{1}\left(C_{p}\right) \text { for some } C_{p} \in \mathscr{C} \text {, } \\
& \text { diam st } h_{2} g\left(C_{q}\right)<\operatorname{diam~st~} C_{q}+\delta<\varepsilon / 3+\varepsilon / 12=5 \varepsilon / 12 \text {, } \\
& h_{2} g h_{1}^{-1}\left(E_{i}\right) \subset \operatorname{Int} h_{2} g\left(C_{p}\right), \quad \text { and } \\
& \rho\left(C_{p}, C_{q}\right) \leqq \rho\left(h_{1}\left(C_{p}\right), C_{p}\right)+\rho\left(h_{1}\left(C_{p}\right), h_{2} g\left(C_{q}\right)\right) \\
& +\rho\left(h_{2} g\left(C_{q}\right), C_{q}\right) \\
& <\delta+0+\delta=2 \delta \text {. }
\end{aligned}
$$

Suppose $C_{p} \cap C_{q}=\varnothing$. Then by (1) $\rho\left(C_{p}, C_{q}\right)>4 \delta$, a contradiction to (10). Hence $C_{p} \cap C_{q} \neq \varnothing$ and $C_{p}$ and $C_{q}$ are identical or adjacent cells. By Lemma 10 there is a homeomorphism $h_{3}$ of $h_{2} g(S)$ onto itself such that $h_{3} h_{2} g h_{1}^{-1}\left(E_{i}\right)=D_{i}$ and for each $x \in h_{2} g(S), x$ and $h_{3}(x)$ are either in the same cell $h_{2} g\left(C_{r}\right)$ or in adjacent cells. Thus by (8) $h_{3}$ is a $5 \varepsilon / 12$ homeomorphism. Let $h=h_{3} h_{2} g h_{1}^{-1}$. Then $h$ is the required homeomorphism.

THEOREM 3. If $S$ is a 2-sphere in $E^{3}$ and $V$ is a component of $E^{3}-S$, then $(* *, \varnothing, V)$ is satisfied.

Proof. This follows immediately from Theorem A and Theorem 2. 
Theorem 3 is a strengthened form of Bing's Side Approximation Theorem, Theorem A.

7. Generalization to a 2-manifold in a 3-manifold. Bing has generalized Theorem A to connected 2-manifolds which separate a connected 3-manifold [5, Th. 1.1]. Using this and methods of the preceding sections we get the following form of this generalization. The proof is omitted since except for details it is like the proof of Theorem 1 followed by the proof of Theorem 2. A 2-manifold $M^{2}$ is tame in a 3-manifold $M^{3}$ if there is a triangulation of the 3-manifold such that $M^{2}$ is the closed union of elements of the triangulation of $M^{3}$.

THEOREM 4. If $M^{2}$ is a connected 2-manifold in a connected 3-manifold $M^{3}$ such that $M^{3}-M^{2}=V_{1} \cup V_{2}, V_{1}$ and $V_{2}$ mutually separated, and $f$ is a positive continuous real valued function on $M^{2}$, then there is a homeomorphism $h$ of $M^{2}$ into $M^{3}$, and a locally finite collection of mutually exclusive disks $\left\{E_{i}\right\}$ on $M^{2}$ such that

(i) $\rho(h(x), x)<f(x)$ for each $x \in M^{2}$,

(ii) $h\left(M^{2}\right)$ is tame,

(iii) $h\left(M^{2}-\mathrm{U} \operatorname{Int} E_{i}\right)=h\left(M^{2}\right)-\mathrm{U} \operatorname{Int} h\left(E_{i}\right) \subset V_{1}$,

(iv) $h\left(E_{i}\right) \cap M^{2}=E_{i} \cap h\left(M^{2}\right)=\left(\operatorname{Int} h\left(E_{i}\right)\right) \cap\left(\operatorname{Int} E_{i}\right)$,

and

(v) $\operatorname{diam} E_{i}<$ minimum value of $f$ on $E_{i}$.

A closely related result, a variant of a theorem of Bing [3, Th. 18], also holds.

Theorem 5. If $U$ is an open subset of a 2-sphere $S$ in $E^{3}, V$ is a component of $E^{3}-S$, and $f$ is a positive continuous real valued function on $U$, then there is a homeomorphism $h$ of $S$ into $E^{3}$ and a locally finite collection of mutually exclusive disks $\left\{E_{i}\right\}$ in $U$ such that

(i ) $h(x)=x$ for each $x \in S-U$,

(ii) $\rho(h(x), x)<f(x)$ for each $x \in U$,

(iii) $h(U)$ is locally polyhedral,

(iv) $h\left(U-U \operatorname{Int} E_{i}\right)=h(U)-U \operatorname{Int} h\left(E_{i}\right) \subset V$,

(v) $U-U$ Int $E_{i} \subset E^{3}-h(S \cup V)$,

(vi) $\operatorname{diam} E_{i}<$ minimum value of $f$ on $E_{i}$, and

(vii) $h\left(E_{i}\right) \cap S=h(S) \cap E_{i}=\left(\operatorname{Int} h\left(E_{i}\right)\right) \cap\left(\operatorname{Int} E_{i}\right)$.

8. Approximation of a 2-sphere by a singular 2-sphere. To prove the invariance of $(* *, F, V)$ under homeomorphisms of $S \cup V$ into $E^{3}$ it is convenient first to prove the following theorem. 
THeOREM 6. If $F$ is a closed subset of a 2-sphere $S$ in $E^{3}$ and $V$ is a component of $E^{3}-S$, then $(* *, F, V)$ is satisfied if and only if for each $\varepsilon>0$ there exist a finite collection of mutually exclusive $\varepsilon$-disks $\left\{E_{1}, E_{2}, \cdots, E_{n}\right\}$ on $S$ and an $\varepsilon$-mapping $g$ of $S$ into (U Int $\left.E_{i}\right) \cup V$ such that

(i) each singularity of $g(S)$ is contained in (U Int $E_{i}$ ),

(ii) $g\left(S-\cup \operatorname{Int} E_{i}\right) \subset V$,

(iii) $g\left(E_{i}\right) \cap S=g(S) \cap E_{i}=g\left(E_{i}\right) \cap \operatorname{Int} E_{i}$, and

(iv) $\left(\bigcup E_{i}\right) \cap F=\varnothing$.

Proof. Suppose, $(* *, V, F)$ is satisfied. Then apply the Tietze Extension Theorem as in the proof of Theorem 1 to obtain the mapping $g$ required in the theorem. To prove the converse, follow a procedure as in the proof of Theorem 1 to apply Dehn's Lemma to each of the singular disks $g\left(E_{i}\right)$ so that an approximating sphere and then an approximating homeomorphism $h$ of $S$ onto this sphere are obtained. Here, of course, we need a form of Dehn's Lemma for nonpiecewise linear maps that can be obtained using one of Bing's approximation theorems [1, Th. 7]. Now $h(S)$ satisfies all of the requirements of property $(* *, F, V)$ except the requirement that $h(S)$ be polyhedral. Bing's approximation theorem [1, Th. 1] can be used to complete the proof.

9. Invariance of $(*, F, V)$ under homeomorphisms of $S \cup V$ into $E^{3}$. It is shown in this section that $(* *, F, V)$ is invariant under homeomorphisms of $S \cup V$ into $E^{3}$. It follows then from Theorem 2 that $(*, F, V)$ is invariant.

THEOREM 7. If $F$ is a closed subset of a 2-sphere $S$ in $E^{3}, V$ is a component of $E^{3}-S,(* *, F, V)$ is satisfied, and $h$ is a homeomorphism of $S \cup V$ into $E^{3}$, then $(* *, h(F), h(V))$ is satisfied.

Proof. Consider first the case in which $V=\operatorname{Int} S$. Let $\varepsilon>0$. There is a $\delta>0$ such that the image $h(X)$ of each $\delta$-subset $X$ of $S \cup \operatorname{Int} S$ is an $\varepsilon / 3$-subset of $h(S \cup \operatorname{Int} S)$. By Theorem 6 there exist a finite collection of $\delta$-disks $\left\{E_{1}, E_{2}, \cdots, E_{n}\right\}$ on $S$ and a $\delta$-mapping $f$ of $S$ into $\left(\bigcup \operatorname{Int} E_{i}\right) \cup \operatorname{Int} S$ such that each singularity of $f(S)$ is contained in (UInt $E_{i}$ ),

$$
\begin{aligned}
& f\left(S-\mathrm{U} \operatorname{Int} E_{i}\right) \subset \operatorname{Int} S, \\
& f\left(E_{i}\right) \cap S=f(S) \cap E_{i}=f\left(E_{i}\right) \cap\left(\operatorname{Int} E_{i}\right), \quad \text { and } \\
& \left(\mathbf{U} E_{i}\right) \cap F=\varnothing \text {. }
\end{aligned}
$$


Consider any $x \in h(S)$. Then $\rho\left(h^{-1}(x), f h^{-1}(x)\right)<\delta$. Hence

$$
\rho\left(x, h f h^{-1}(x)\right)<\varepsilon / 3 \text {. }
$$

Also diam $h\left(E_{i}\right)<\varepsilon / 3$ and it follows that diam $h f h^{-1}\left(h\left(E_{i}\right)\right)<\varepsilon$. Now for the sphere $h(S)$, the closed subset $h(F)$, and the collection of disks $\left\{h\left(E_{1}\right), h\left(E_{2}\right), \cdots, h\left(E_{n}\right)\right\}$, the mapping $h f h^{-1}$ satisfies the requirements of the mapping $g$ in Theorem 6 . Hence it follows from Theorem 6 that $(* *, h(F), h(\operatorname{Int} S))$ is satisfied. A similar proof can be used for the case in which $V=\operatorname{Ext} S$ by enclosing $S$ in the interior of a large 3-cell $K$, so that $h$ would be uniformly continuous on $K-\operatorname{Int} S$.

10. Embeddings of crumpled cubes in $E^{3}$. A crumpled cube is a space which is homeomorphic to a 2-sphere plus its interior in $E^{3}$. In this section we give some theorems on the preservation of tameness and half tameness of subsets of the boundary of a crumpled cube under re-embeddings of the crumpled cube in $E^{3}$.

Loveland has shown that if $(*, F, V)$ is satisfied for a closed subset $F$ of a 2 -sphere $S$ in $E^{3}$ with complementary domain $V$, then $F$ is half tame [11] and if $(*, F$, Int $S)$ and $(*, F$, Ext $S)$ are both satisfied, then $F$ is tame [10]. It is clear that if a 2-sphere $S$ is tame from $V$, then $(*, F, V)$ is satisfied for each closed subset $F$ of $S$. The next two theorems follow from these results and Theorem 7 .

Theorem 8. If $F$ is a closed subset of a 2-sphere $S$ in $E^{3}, V$ is a component of $E^{3}-S,(*, F, V)$ is satisfied, and $h$ is a homeomorphism of $S \cup V$ into $E^{3}$, then $h(F)$ is half tame.

THEOREM 9. If $F$ is a closed subset of a 2-sphere $S$ in $E^{3}, V$ is a component of $E^{3}-S,(*, F, V)$ is satisfied, and $h$ is a homeomorphism of $S \cup V$ into $E^{3}$ such that $h(S)$ is tame from $E^{3}-h(S \cup V)$, then $h(F)$ is tame.

Hosay [7] and Lininger [9] have independently shown that if $S$ is a 2-sphere in $E^{3}, V$ is a component of $E^{3}-S$, and $\varepsilon>0$, then there is an $\varepsilon$-homeomorphism $h$ of $S \cup V$ into $E^{3}$ such that $h(S)$ is tame from $E^{3}-h(S \cup V)$. The next theorem follows from this and Theorem 9 .

THEOREM 10. If $F$ is a closed subset of a 2-sphere $S$ in $E^{3}, V$ is a component of $E^{3}-S,(*, F, V)$ is satisfied, and $\varepsilon>0$, then there is an e-homeomorphism $h$ of $S \cup V$ into $E^{3}$ such that $h(F)$ is tame.

As pointed out by Loveland in [10] it is known from results of Bing [4] and methods of Gillman [6] that if $F$ is a finite union of 
tame finite graphs and tame Sierpiński curves on a 2-sphere $S$ in $E^{3}$, then $(*, F$, Int $S)$ and $(*, F$, Ext $S)$ are satisfied. Theorem 9 and this result imply the following theorem.

THEOREM 11. If $F$ is a finite union of tame finite graphs and tame Sierpinski curves on a 2-sphere $S$ in $E^{3}, V$ is a component of $E^{3}-S$, and $h$ is a homeomorphism of $S \cup V$ into $E^{3}$ such that $h(S)$ is tame from $E^{3}-h(S \cup V)$, then $h(F)$ is tame.

Question. Can Theorem 11 be generalized to tame closed subsets of a 2-sphere with no degenerate components? If it were known that $(*, F, V)$ holds for such subsets of a 2-sphere, then the theorem would generalize immediately.

\section{REFERENCES}

1. R. H. Bing, Approximating surfaces with polyhedral ones, Ann. of Math. (2) 65 (1957), 456-483.

2. - A surface is tame if its complement is 1-ULC, Trans. Amer. Math. Soc. 101 (1961), 294-305.

3. — Approximating surfaces from the side, Ann. of Math. (2) 77 (1963), 145192.

4. —, Pushing a sphere into its complement, Michigan Math. J. 11 (1964), 33-45.

5. - Improving the side approximation theorem, Trans. Amer. Math. Soc. 116 (1965), 511-525.

6. David S. Gillman, Side approximation missing an arc, Amer. J. Math. 85 (1963), 459-476.

7. Norman Hosay, The sum of a real cube and a crumpled cube is $S^{3}$, Abstract 60717, Notices Amer. Math. Soc. 10 (1963), 668.

8. Hurewicz and Wallman, Dimension Theory, Princeton University Press, Princeton, New Jersey, 1948.

9. Lloyd L. Lininger, Some results on crumpled cubes, Trans. Amer. Math. Soc. 118 (1965), 534-540.

10. L. D. Loveland, Tame subsets of spheres in $E^{3}$, Pacific J. Math. 19 (1966), 489-517. 11. _ Sufficient conditions for a closed set to lie on the boundary of a 3-cell (to be submitted)

12. C. D. Papakyriakopoulos, On Dehn's Lemma and the asphericity of knots, Ann. of Math. (2) 66 (1957), 1-26.

13. R. L. Wilder, Topology of Manifolds, Amer. Math. Soc. Colloq. Publ. Vol. 32, Amer. Math. Soc., Providence, R. I., 1949.

Received June 3, 1966. This paper is part of the author's Ph. D. thesis written under the direction of Professor C. E. Burgess at the University of Utah. Work was begun on this paper while the author was a National Science Foundation Science Faculty Fellow. 



\section{PACIFIC JOURNAL OF MATHEMATICS}

\section{EDITORS}

\section{H. SAMELSON}

Stanford University

Stanford, California

J. P. JANS

University of Washington

Seattle, Washington 98105

\section{J. DugundJI}

University of Southern California Los Angeles, California 90007

RICHARD ARENS

University of California

Los Angeles, California 90024

\section{ASSOCIATE EDITORS}

E. F. BECKENBACH
B. H. NeUmanN

\section{SUPPORTING INSTITUTIONS}

UNIVERSITY OF BRITISH COLUMBIA CALIFORNIA INSTITUTE OF TECHNOLOGY

UNIVERSITY OF CALIFORNIA

MONTANA STATE UNIVERSITY

UNIVERSITY OF NEVADA

NEW MEXICO STATE UNIVERSITY

OREGON STATE UNIVERSITY

UNIVERSITY OF OREGON

OSAKA UNIVERSITY

UNIVERSITY OF SOUTHERN CALIFORNIA

\author{
STANFORD UNIVERSITY \\ UNIVERSITY OF TOKYO \\ UNIVERSITY OF UTAH \\ WASHINGTON STATE UNIVERSITY \\ UNIVERSITY OF WASHINGTON \\ AMERICAN MATHEMATICAL SOCIETY \\ CHEVRON RESEARCH CORPORATION \\ TRW SYSTEMS \\ NAVAL ORDNANCE TEST STATION
}

Mathematical papers intended for publication in the Pacific Journal of Mathematics should be typewritten (double spaced). The first paragraph or two must be capable of being used separately as a synopsis of the entire paper. It should not contain references to the bibliography. Manuscripts may be sent to any one of the four editors. All other communications to the editors should be addressed to the managing editor, Richard Arens at the University of California, Los Angeles, California 90024.

50 reprints per author of each article are furnished free of charge; additional copies may be obtained at cost in multiples of 50 .

The Pacific Journal of Mathematics is published monthly. Effective with Volume 16 the price per volume ( 3 numbers) is $\$ 8.00$; single issues, $\$ 3.00$. Special price for current issues to individual faculty members of supporting institutions and to individual members of the American Mathematical Society: $\$ 4.00$ per volume; single issues $\$ 1.50$. Back numbers are available.

Subscriptions, orders for back numbers, and changes of address should be sent to Pacific Journal of Mathematics, 103 Highland Boulevard, Berkeley 8, California.

Printed at Kokusai Bunken Insatsusha (International Academic Printing Co., Ltd.), 7-17, Fujimi 2-chome, Chiyoda-ku, Tokyo, Japan.

\section{PUBLISHED BY PACIFIC JOURNAL OF MATHEMATICS, A NON-PROFIT CORPORATION}

The Supporting Institutions listed above contribute to the cost of publication of this Journal, but they are not owners or publishers and have no responsibility for its content or policies. 


\section{Pacific Journal of Mathematics}

\section{Vol. 22, No. $2 \quad$ February, 1967}

Paul Frank Baum, Local isomorphism of compact connected Lie groups ....

Lowell Wayne Beineke, Frank Harary and Michael David Plummer, On the

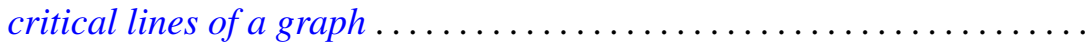

Larry Eugene Bobisud, On the behavior of the solution of the telegraphist's equation for large velocities .......................... 213

Richard Thomas Bumby, Irreducible integers in Galois extensions . . . . . . 221

Chong-Yun Chao, A nonimbedding theorem of nilpotent Lie algebras ..... 231

Peter Crawley, Abelian p-groups determined by their Ulm sequences ...... 235

Bernard Russel Gelbaum, Tensor products of group algebras ........... 241

Newton Seymour Hawley, Weierstrass points of plane domains .......... 251

Paul Daniel Hill, On quasi-isomorphic invariants of primary groups . . . . . 257

Melvyn Klein, Estimates for the transfinite diameter with applications to confomral mapping ................................ 267

Frederick M. Lister, Simplifying intersections of disks in Bing's side approximation theorem ............................. 281

Charles Wisson McArthur, On a theorem of Orlicz and Pettis ........... 297

Harry Wright McLaughlin and Frederic Thomas Metcalf, An inequality for

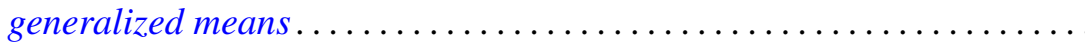

Daniel Russell McMillan, Jr., Some topological properties of piercing

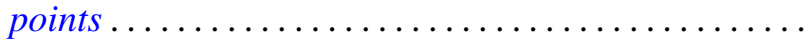

Peter Don Morris and Daniel Eliot Wulbert, Functional representation of topological algebras .

Roger Wolcott Richardson, Jr., On the rigidity of semi-direct products of Lie algebras..................................

Jack Segal and Edward Sandusky Thomas, Jr., Isomorphic

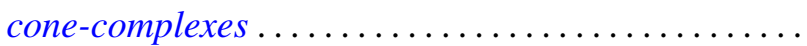

Richard R. Tucker, The $\delta^{2}$-process and related topics.... 